\title{
BMJ Open Anticoagulation regimens during pregnancy in patients with mechanical heart valves: a protocol for a systematic review and network meta-analysis
}

\author{
Shiwei He, ${ }^{1}$ Yue Zou, ${ }^{1}$ Juan Li, ${ }^{2}$ Jumei Liu, ${ }^{2}$ Li Zhao, ${ }^{3}$ Hua Yang, ${ }^{4}$ Zhiying Su, ${ }^{4}$ \\ Huiming Ye (D) ${ }^{1,2,3}$
}

To cite: He S, Zou Y, Li J, et al. Anticoagulation regimens during pregnancy in patients with mechanical heart valves: a protocol for a systematic review and network meta-analysis. BMJ Open 2020;10:e033917. doi:10.1136/ bmjopen-2019-033917

\section{- Prepublication history and} additional material for this paper are available online. To view these files, please visit the journal online (http://dx.doi. org/10.1136/bmjopen-2019033917).

Received 29 August 2019 Revised 21 December 2019 Accepted 23 December 2019

Check for updates

(C) Author(s) (or their employer(s)) 2020. Re-use permitted under CC BY-NC. No commercial re-use. See rights and permissions. Published by BMJ.

${ }^{1}$ School of Public Health, Xiamen University, Xiamen, China

${ }^{2}$ Department of Clinical Laboratory, Women and Children's Hospital, School of Medicine, Xiamen University, Xiamen, China

${ }^{3}$ School of Medicine, Xiamen University, Xiamen, China ${ }^{4}$ Department of Obstetrics, Women and Children's Hospital, School of Medicine, Xiamen University, Xiamen, China

Correspondence to Dr. Huiming Ye; yehuiming@xmu.edu.cn

Professor Zhiying Su; dyyyszy@126.com

\section{ABSTRACT}

Introduction Pregnancy in patients with mechanical heart valves (MHVs) is associated with high maternal complications and fetal complications.Anticoagulation treatments serve to decrease their venous clotting risk Although some anticoagulation regimens have been used for patients during pregnancy with MHVs, no one is definitively superior among different regimens in recent studies. For a better understanding of the clinical treatment which anticoagulation regimen is more effective and safer during the pregnancy in patients with MHVs, a Bayesian network meta-analysis is necessary. Methods and analysis This protocol has been reported according to the Preferred Reporting Items for Systematic Reviews and Meta-Analyses Protocols. Related studies until April 2019 will be searched in the following databases: PubMed, Embase,SinoMed and the using the OVID interface to search for evidence-based medicine reviews. A clinical trial registry (www.ClinicalTrials.gov) was also searched for unpublished trials. Both experimental studies (randomised clinical trials) and observational studies (cohort studies, case-control studies and case series studies) will be included in this study. Quality assessment will be conducted using Cochrane Collaboration's tool or Newcastle-Ottawa Scale based on their study designs. The primary outcomes of interest will be the frequencies of serious maternal and fetal events. The additional outcomes of interest will be adverse maternal events, mode of delivery and adverse fetal events. Pairwise and network meta-analysis will be conducted using R (V.3.4.4, R Foundation for Statistical Computing, Vienna, Austria) and Stata (V.14, StataCorp). The ranking probabilities will be estimated at each possible rank for each anticoagulation regimen using the surface under the cumulative ranking curve. Statistical inconsistency assessment, subgroup analysis, sensitivity analysis and publication bias assessment will be performed.

Ethics and dissemination Either ethics approval or patient consent is not necessary, because this study will be based on literature. The results of this study will be published in a peer-reviewed journal.

PROSPERO registration number CRD42019130659

\section{INTRODUCTION}

Although mechanical heart valves (MHVs) have excellent durability and haemodynamic
Strengths and limitations of this study

- This study will be the first Bayesian network metaanalysis (NMA) that evaluates the comparative effects of multiple anticoagulation regimens in patients during pregnancy with mechanical heart valves.

- This study will include both experimental studies and observational studies in this study to strengthen the statistical power.

- This study will use the Grade of Recommendations Assessment, Development and Evaluation system to assess the quality of included studies.

- Most of the observational studies in this study will be retrospective studies, which will increase the risk of inferior quality of the results.

- The number of included studies may be relatively small which will reduce the ability to explore heterogeneity, conduct meta regression and even perform NMA.

profiles, they are thrombogenic, and the patients with a MHV require lifelong anticoagulation to prevent thromboembolic complications. ${ }^{12}$ Moreover, normal pregnancy is accompanied by changes in haemostasis that produce a hypercoagulable state. ${ }^{3}$ As a result, pregnancy in a woman with a MHV is associated with high maternal complications (eg, thromboembolic complications, heart failure, arrhythmias and bleeding, etc) and fetal complications (eg, fetal wastage, preterm birth, low birth weight and teratogenicity, etc) ${ }^{45}$ Furthermore, the incidence and prevalence of cardiothoracic disease continue to increase globally. ${ }^{6}$ It means that a large number of MHVs have been developed and are implanted worldwide, many in women of childbearing age. ${ }^{7}$ Cardiac disease, for example, previous valve replacement because of rheumatic heart disease, is emerging as the most important indirect cause of maternal death globally. ${ }^{89}$ 
Although women cannot alter the physiological changes that occur naturally during pregnancy, anticoagulation treatments serve to decrease their venous clotting risk. ${ }^{10}$ In recent guidelines, vitamin $\mathrm{K}$ antagonists (VKA), heparin (including low-molecular-weight heparin (LMWH) and unfractionated heparin (UFH)) and sequential treatments are recommended to take into the anticoagulation regimens during pregnancy in patients with MHVs. ${ }^{11} 12$ However, the use of VKA such as warfarin during pregnancy carries the potential for serious risks of fetal embryopathy. ${ }^{13}{ }^{14}$ Neither UFH nor LMWH crosses the placenta, and therefore, are considered safe for mother and fetus, but in the previous literature, ${ }^{15}$ some circumstances included the presence of heparin resistance and heparin allergy manifesting limited their use; moreover, heparin (specifically UFH) was associated with an increased thrombotic risk. Sequential treatments refer to the use of VKAs in the second and third trimesters and heparin in the first trimester and also in the peripartum period, to mitigate the VKA-related risks previously alluded to. ${ }^{16}$ Although the use of this regimen could avoid the risk of warfarin embryopathy and would minimise the time off VKAs and perhaps be associated with a more favourable maternal risk profile, it would not prevent the fetal bleeding complications. ${ }^{16}$ The evidence related to the safety of new oral anticoagulant (NOACs) in pregnant women and in those planning pregnancy is scarce; therefore, NOACs currently have no place during pregnancy. ${ }^{17} 18$

Several regimens have been recommended and advised by different guidelines; however,recently study does not suggest that one regimen is definitively superior. ${ }^{19}$ Thus, the evidence for the anticoagulation regimens comparisons during pregnancy in patients with MHVs consists of direct head-to-head comparisons of treatments in randomised controlled trials (RCTs) and observational studies. Although, several meta-analyses related to this research topic have been published previously, all of them are traditional pairwise meta-analyses, which included some obvious limitations that need to be urgently improved. ${ }^{13} 20-22$ First, synthesising evidence using the traditional pairwise meta-analyses would not allow for the inclusion of data from treatments (eg, the comparations of different sequential treatments) that have not been compared head-to-head in Xu et al.'s, D'Souza et $a l$.'s, Steinberg et al.'s and Chan $e t a l$ 's studies. ${ }^{13-23}$ The results from indirect combined with direct evidence using network meta-analysis (NMA) allows for simultaneous consideration of the relative effectiveness and safety of all available anticoagulation treatments. ${ }^{23}$ Furthermore, an NMA can estimate the rank of these treatments. ${ }^{23}{ }^{24}$ Second, some high-quality and latest studies (one $\mathrm{RCT}^{25}$ and nine observational studies) ${ }^{26-34}$ in recent years were not included in these studies, which reduced trustworthiness and statistical power of these studies. Finally, some subgroups of anticoagulation treatments (eg, different VKAs and heparin doses, different combinations of sequential treatments, and type, location and number of MHVs, etc) were not considered in these studies, which led to the lack of results of effectiveness and safety by comparing these subgroups. These research gaps pose a urgently practical challenge to clinicians for choosing a suitable anticoagulation regimen because a direct comparison is rarely seen or not available for many anticoagulation regimens. Therefore, to address the challenge of clinicians to determine which anticoagulation regimen is more effective and safer during pregnancy in patients with MHVs, a Bayesian NMA is necessary.

\section{OBJECTIVE}

The objectives of this study are to synthesise the available evidence on anticoagulation regimens during pregnancy in patients with MHVs, to estimate the treatment effects among direct and indirect treatment comparisons, and to determine which anticoagulation regimen is more effective and safer using a Bayesian NMA.

\section{METHODS AND DESIGN \\ Design}

This protocol has been reported according to the Preferred Reporting Items for Systematic Reviews and Meta-Analyses (PRISMA) Protocols ${ }^{35}{ }^{36}$ (see online supplementary file 1). The study will be conducted and reported according to PRISMA Extension for NMAs of healthcare interventions guidelines. ${ }^{37}$ The Bayesian NMA will be used in this study.

\section{Patient and public involvement}

No patients or the public were involved in this study. However, the results will be disseminated to patients during the pregnancy with MHVs receiving anticoagulation treatment.

\section{Information source and search strategy}

PubMed <to 3 April 2019>, Embase <to 3 April 2019 >, SinoMed <to April 2019> and the using the OVID interface, to search for evidence-based medicine reviews: Cochrane Database of Systematic Reviews <2005 to 27 March 2019>, ACP Journal Club<1991 to March 2019>, Database of Abstracts of Reviews of Effects $<1$ st Quarter 2016>, Cochrane Clinical Answers <March 2019>, Cochrane Central Register of Controlled Trials $<$ March 2019>, Cochrane Methodology Register $<3$ rd Quarter 2012>, Health Technology Assessment $<4$ th Quarter 2016> and NHS Economic Evaluation Database $<1$ st Quarter 2016>. Clinical trial registries (such as www.ClinicalTrials.gov) were also searched for unpublished trials.

In addition, references of included studies and narrative reviews were considered for additional potential studies. No limitations will be imposed on publication status, language of dissemination, duration of study follow-up or period of study conduct. The search strategy is shown in online supplementary file 2. 


\section{Eligibility criteria}

\section{Types of participants}

This study will include pregnant patients (conception to 6 months postpregnancy regardless of the outcome of pregnancy) who require long-term anticoagulation with MHVs. Non-pregnant patients and pregnant patients with bioprosthetic valves not requiring anticoagulation will not be included.

\section{Types of interventions}

This study will include studies comparing at least two different interventions among the following interventions: (1) dose-adjusted VKA throughout pregnancy, (2) dose-adjusted LMWH throughout pregnancy, (3) doseadjusted UFH throughout pregnancy, (4) dose-adjusted LMWH for the first trimester, followed by a VKA for the remainder (LMWH and VKA), (5) dose-adjusted UFH for the first trimester, followed by a VKA for the remainder (UFH and VKA) and (6) other antagonists or placebo, including acetylsalicylic acid, NOACs, fondaparinux and argatroban.

\section{Type of outcomes}

The primary outcomes of interest will be the frequencies of serious maternal and fetal events. Maternal events of interest will include all thromboembolic complications including valve thrombosis, major bleeding and maternal death. Fetal outcomes will include live births, anticoagulant-related fetal adverse events (including warfarin embryopathy, neurological sequelae related to VKA, other congenital abnormalities) and fetal wastage (including spontaneous abortions (fetal loss $<20$ weeks), therapeutic abortions, stillbirths (fetal loss $>20$ weeks), fetal loss (where definitions of miscarriage/stillbirth are uncertain) and neonatal death (death within the first 28 days of life)). The additional outcomes of interest will be adverse maternal events, mode of delivery and adverse fetal events. Maternal adverse events will include cardiac events including new maternal arrhythmia, infective endocarditis, valve deterioration, myocardial infarction, pregnancy hypertension, heart failure and other adverse drug effects from anticoagulation. Mode of delivery will be either caesarean section or vaginal birth. Adverse fetal events will include prematurity, small for gestational age infants, preterm births under 37 weeks and infant admission to neonatal intensive care unit. The types of outcomes were chosen referred to previous investigation. ${ }^{1320-22}$

\section{Types of studies}

We will include experimental studies (RCTs) and observational studies (cohort studies, case-control studies and case series studies).

\section{Study selection}

To assess study eligibility, all title/abstracts and full-text articles will be independently screened by two reviewers ( $\mathrm{SH}$ and $\mathrm{YZ}$ ) and disagreements will be resolved by a third reviewer (JL). If necessary, methodological experts will be consulted to reach consensus. Eligible articles will be selected according to inclusion criteria. If studies have duplicate data, only the study with larger sample size and longer follow-up time will be included.

\section{Data extraction}

Data will be extracted by three reviewers ( $\mathrm{SH}$, JL and YZ) based on an extraction form, independently and in duplicate, using Excel software regarding: (1) study information (author, publication year, sample size, duration of study, etc), (2) participant characteristics (age; type, location and number of MHVs; time since valve repair; The New York Heart Association class and cardiac status at the onset of pregnancy; medical and obstetric comorbidities; details of labour and delivery, etc), (3) intervention characteristics (details of the anticoagulation regimens including the name of anticoagulants, duration of treatment, rate of compliance with treatment, details on adjustment of anticoagulation and route of administration, etc), (4) reported outcomes (outcome data for the main outcomes and additional outcomes of interest). The types of data were chosen referred to previous investigation. ${ }^{13}{ }^{20-22}$ Missing data will be requested from study authors. Discrepancies will be resolved by consensus and when necessary, consultation with an expert on the investigative team.

\section{Risk of bias (quality) assessment}

The risk of bias of the included studies will be assessed using the Cochrane risk of bias tool and Newcastle-Ottawa scale for randomised controlled trials and observational studies, respectively. ${ }^{38}{ }^{39}$ Two reviewers (SH and YZ) will conduct quality assessment independently and any disagreement will be solved by discussion with another author (JL).

\section{Data synthesis}

When quantitative analysis cannot be conducted, we will narratively describe the results. If quantitative analysis is feasible, all of the following statistical analyses will be conducted using R (V.3.4.4, R Foundation for Statistical Computing, Vienna, Austria) and Stata (V.14, StataCorp). And, the binary outcomes will be presented as ORs with $95 \%$ CIs.

\section{Direct comparisons of interventions}

All the direct comparisons will be performed using the DerSimonian-Laird method and random effects model. ${ }^{40}$ Q-test and I-squared statistic will be used to assess heterogeneity levels, as a measure of the proportion of the overall variation that is attributable to between study heterogeneity. ${ }^{41}$

\section{Indirect and mixed comparisons of interventions}

A random-effects NMA within a Bayesian framework will then be applied. ${ }^{42}{ }^{43}$ Interactions among all included studies will be shown in the network geometry, and the contribution plot for the network will show the contributions of direct comparisons. ${ }^{44}$ We will estimate the ranking probabilities at each possible rank for each 
anticoagulation regimen using the surface under the cumulative ranking curve. ${ }^{45}$

\section{Assessment of inconsistency}

To check the assumption of consistency in the entire analytical network, a design-by-treatment approach will be used. ${ }^{46} \mathrm{~A}$ loop-specific approach will be applied to evaluate the presence of inconsistency locally in each closed loop. ${ }^{47}$ And, the node-splitting method will be used to assess the inconsistency of the model by separating evidence on particular comparisons into direct and indirect evidence. ${ }^{48}$

\section{Subgroup analysis and sensitivity analysis}

If there are sufficient data, we will assess whether the results have been impacted by study characteristics, subgroup analyses may be conducted according to age group, sample size, quality of study, duration of treatment and timing of medication usage in pregnancy. And, a sensitivity analysis will also be conducted to validate the robustness of the results by excluding each study.

\section{Publication bias}

Publication bias will be assessed by visually examining the comparison-adjusted funnel plot asymmetry and Egger's regression test in the results between small and large studies. ${ }^{49}$

\section{Quality of evidence}

We will use the Grade of Recommendation Assessment, Development and Evaluation (GRADE) approach to appraise the quality of direct and indirect evidence. ${ }^{50}$

\section{DISCUSSION}

This study will first determine which anticoagulation regimen during pregnancy in patients with MHVs is more effective and safer using a Bayesian NMA. We expect that our findings will inform clinicians, patients and guideline developers the best available evidence on the efficacy and safety of different anticoagulation regimens during pregnancy in patients with MHVs, which will help both clinical practice and study design in the future. We will include both experimental studies and observational studies in this study to strengthen the statistical power, because the number of related experimental studies, such as RCTs, is still small. Moreover, we will use GRADE to assess the quality of included studies. However, most of the observational studies will be retrospective studies in our study, inclusion of those studies will increase the risk of inferior quality of the results. Furthermore, different types of study will generate potentially heterogeneity which may influence the results of this study.

\section{ETHICS AND DISSEMINATION}

\section{Publication plan}

This protocol has been successfully registered on PROSPERO. The final results of this study will be published in a peer-reviewed journal.

Contributors $\mathrm{SH}$ and $\mathrm{HY}$ are responsible for the conception of the protocol. $\mathrm{SH}$, YZ, JLi, JLiu, LZ and HY were involved in the design of this protocol. SH, YZ,JLi and JLiu tested the feasibility of this protocol. SH, YZ and JLi wrote the original draft. $\mathrm{HY}, \mathrm{ZS}$ and HY reviewed the draft and approved the final manuscript as submitted. All authors contributed to the development of the selection criteria. All authors read, provided feedback and approved the final manuscript as submitted.

Funding This study was supported by the National Natural Science Foundation of China $(81472031,81101331)$ and Foundation of Fujian Provincial Health System for Outstanding Young Doctors (2015-WZK-ZD-32) and Xiamen Youth Innovation Talents Project (2015-A-03).

Competing interests None declared.

Patient consent for publication Not required.

Provenance and peer review Not commissioned; externally peer reviewed.

Open access This is an open access article distributed in accordance with the Creative Commons Attribution Non Commercial (CC BY-NC 4.0) license, which permits others to distribute, remix, adapt, build upon this work non-commercially, and license their derivative works on different terms, provided the original work is properly cited, appropriate credit is given, any changes made indicated, and the use is non-commercial. See: http://creativecommons.org/licenses/by-nc/4.0/.

\section{ORCID iD}

Huiming Ye http://orcid.org/0000-0003-2069-3644

\section{REFERENCES}

1 Castellano JM, Narayan RL, Vaishnava P, et al. Anticoagulation during pregnancy in patients with a prosthetic heart valve. Nat Rev Cardiol 2012;9:415-24.

2 JCJ S, Davidson MJ, Lamy A, et al. Antithrombotic management of patients with prosthetic heart valves: current evidence and future trends. The Lancet 2009;374:565-76.

3 Meah VL, Cockcroft JR, Backx K, et al. Cardiac output and related haemodynamics during pregnancy: a series of meta-analyses. Heart 2016;102:518-26.

4 Bhagra CJ, D'Souza R, Silversides CK. Valvular heart disease and pregnancy Part II: management of prosthetic valves. Heart 2017;103:244-52.

5 Elkayam U. Anticoagulation Therapy for Pregnant Women With Mechanical Prosthetic Heart Valves: How to Improve Safety? J Am Coll Cardiol 2017;69:2692-5.

6 Pezzella AT. Global aspects of cardiothoracic surgery with focus on developing countries. Asian Cardiovasc Thorac Ann 2010;18:299-310.

7 Sliwa K, Johnson MR, Zilla P, et al. Management of valvular disease in pregnancy: a global perspective. Eur Heart J 2015;36:1078-89.

8 Kassebaum NJ, Barber RM, Bhutta ZA, et al. Global, regional, and national levels of maternal mortality, 1990-2015: a systematic analysis for the global burden of disease study 2015. Lancet 2016;388:1775-812.

9 Sliwa K, Anthony J. Risk assessment for pregnancy with cardiac disease-a global perspective. Eur J Heart Fail 2016;18:534-6.

10 Fogerty AE. Challenges of anticoagulation therapy in pregnancy. Curr Treat Options Cardiovasc Med 2017;19.

11 Nishimura RA, Otto CM, Bonow RO, et al. 2014 AHA/ACC guideline for the management of patients with valvular heart disease. $J$ Thorac Cardiovasc Surg 2014;148:e1-132.

12 Regitz-Zagrosek V, Roos-Hesselink JW, Bauersachs J, et al. 2018 ESC guidelines for the management of cardiovascular diseases during pregnancy. Eur Heart J 2018;39:3165-241.

13 Chan WS, Anand S, Ginsberg JS. Anticoagulation of pregnant women with mechanical heart valves: a systematic review of the literature. Arch Intern Med 2000;160:191-6.

14 Walfisch A, Koren G. The "warfarin window" in pregnancy: the importance of half-life. J Obstet Gynaecol Can 2010;32:988-9.

15 James AH. Prevention and management of thromboembolism in pregnancy when heparins are not an option. Clin Obstet Gynecol 2018;61:1-34. 
16 D'Souza R, Silversides C, McLintock C. Optimal anticoagulation for pregnant women with mechanical heart valves. Semin Thromb Hemost 2016;42:798-804

17 Cohen H, Arachchillage DR, Middeldorp S, et al. Management of direct oral anticoagulants in women of childbearing potential: guidance from the SSC of the ISTH. J Thromb Haemost 2016;14:1673-6.

18 Scheres LJJ, Bistervels IM, Middeldorp S. Everything the clinician needs to know about evidence-based anticoagulation in pregnancy. Blood Rev 2019;33:82-97.

19 van Hagen IM, Roos-Hesselink JW, Ruys TPE, et al. Pregnancy in women with a mechanical heart valve data of the European Society of cardiology registry of pregnancy and cardiac disease (ROPAC) Circulation 2015;132:132-42.

20 D'Souza R, Ostro J, Shah PS, et al. Anticoagulation for pregnant women with mechanical heart valves: a systematic review and metaanalysis. Eur Heart J 2017;38:1509-16.

21 Steinberg ZL, Dominguez-Islas CP, Otto CM, et al. Maternal and Fetal Outcomes of Anticoagulation in Pregnant Women With Mechanical Heart Valves. J Am Coll Cardiol 2017;69:2681-91.

22 Xu Z, Fan J, Luo X, et al. Anticoagulation regimens during pregnancy in patients with mechanical heart valves: a systematic review and meta-analysis. Can J Cardiol 2016;32:1248.e1-9.

23 Riley RD, Jackson D, Salanti G, et al. Multivariate and network metaanalysis of multiple outcomes and multiple treatments: rationale, concepts, and examples. BMJ-British Medical Journal 2017;358:13.

24 Cameron C, Fireman B, Hutton B, et al. Network meta-analysis incorporating randomized controlled trials and non-randomized comparative cohort studies for assessing the safety and effectiveness of medical treatments: challenges and opportunities. Syst Rev 2015:4:147.

25 Hassouna A, Ammar A, Elnahas Y, et al. Limited dose warfarin throughout pregnancy in high-risk patients with mechanical valves: a randomized clinical trial. The Egyptian Heart Journal 2015;67:115-22.

26 Akyol A, Yaman M, Şahin M, et al. Maternal, fetal outcome, and anticoagulant management in pregnant women with prosthetic heart valves. Clinical and Experimental Obstetrics and Gynecology 2018;45:218-23.

27 Ayad SW, Hassanein MM, Mohamed EA, et al. Maternal and fetal outcomes in pregnant women with a prosthetic mechanical heart valve. Clinical Medicine Insights: Cardiology 2016;10:11-17.

28 Benatta N, Batouche DD. Anticoagulation's problematic during pregnancy in carriers of mechanical heart prostheses. Archives of Cardiovascular Diseases Supplements 2017:9:75-6.

29 Benatta NF, Batouche DD, Djazouli MA. [Anticoagulation's problematic during pregnancy in carriers of mechanical heart prosthesis]. Ann Cardiol Angeiol 2018;67:270-3.

30 Bian C, Qi X, Li L, et al. Anticoagulant management of pregnant women with mechanical heart valve replacement during perioperative period. Arch Gynecol Obstet 2016;293:69-74.

31 Kalcik M, Bayam E, Yesin M, et al. P6047Comparison of different anticoagulation regimens in pregnant patients with mechanical prosthetic heart valves. Eur Heart J 2017;38:1271-2.

32 Khader KAAM, Saad AS, Abdelshafy M. Pregnancy outcome in women with mechanical prosthetic heart valves treated with unfractionated heparin (UFH) or enoxaparin. J Obstet Gynecol India 2016;66:321-6.

33 van Hagen IM, Roos-Hesselink JW, Ruys TPE, et al. Pregnancy in women with a mechanical heart valve. Circulation 2015;132:132-42.

34 Vause S, Clarke B, Tower CL, et al. Pregnancy outcomes in women with mechanical prosthetic heart valves: a prospective descriptive population based study using the United Kingdom obstetric surveillance system (UKOSS) data collection system. BJOG: Int $J$ Obstet Gy 2017;124:1411-9.

35 Moher D, Shamseer L, Clarke M, et al. Preferred reporting items for systematic review and meta-analysis protocols (PRISMA-P) 2015 statement. Syst Rev 2015;4:1.

36 Shamseer L, Moher D, Clarke M, et al. Preferred reporting items for systematic review and meta-analysis protocols (PRISMA-P) 2015 elaboration and explanation. BMJ 2015;349:97647.

37 Hutton B, Salanti G, Caldwell DM, et al. The PRISMA extension statement for reporting of systematic reviews incorporating network meta-analyses of health care interventions: checklist and explanations. Ann Intern Med 2015;162:777-84.

38 Higgins JPT, Altman DG, Gotzsche PC, et al. The Cochrane collaboration's tool for assessing risk of bias in randomised trials. BMJ 2011;343:d5928.

39 Stang A. Critical evaluation of the Newcastle-Ottawa scale for the assessment of the quality of nonrandomized studies in metaanalyses. Eur J Epidemiol 2010;25:603-5.

40 DerSimonian R, Laird N. Meta-Analysis in clinical trials revisited. Contemp Clin Trials 2015;45:139-45.

41 Perleth M, Langer G, Meerpohl JJ, et al. [GRADE guidelines: 7. Rating the quality of evidence - inconsistency]. Z Evid Fortbild Qual Gesundhwes 2012;106:733-44.

42 Dias S, Sutton AJ, Ades AE, et al. Evidence synthesis for decision making 2: a generalized linear modeling framework for pairwise and network meta-analysis of randomized controlled trials. Medical Decision Making 2013;33:607-17.

43 Lu G, Ades AE. Combination of direct and indirect evidence in mixed treatment comparisons. Stat Med 2004:23:3105-24.

44 Salanti G. Ades AE, loannidis JPA. Graphical methods and numerical summaries for presenting results from multiple-treatment metaanalysis: an overview and tutorial. J Clin Epidemiol 2011;64:163-71.

45 Chaimani A, Higgins JPT, Mavridis D, et al. Graphical tools for network meta-analysis in STATA. PLoS One 2013;8:12.

46 White IR, Barrett JK, Jackson D, et al. Consistency and inconsistency in network meta-analysis: model estimation using multivariate metaregression. Res Synth Methods 2012;3:111-25.

47 Veroniki AA, Vasiliadis HS, Higgins JP, et al. Evaluation of inconsistency in networks of interventions. Int $J$ Epidemiol 2013;42:332-45

48 Yu-Kang T. Node-Splitting generalized linear mixed models for evaluation of inconsistency in network meta-analysis. Value Health 2016;19:957-63.

49 Sterne JAC, Sutton AJ, loannidis JPA, et al. Recommendations for examining and interpreting funnel plot asymmetry in meta-analyses of randomised controlled trials. BMJ 2011;343:d4002.

50 Salanti G, Del Giovane C, Chaimani A, et al. Evaluating the quality of evidence from a network meta-analysis. PLoS One 2014;9:e99682. 